\title{
MS14-P38 | Preparation, Spectral, Structural, Thermal and anticancer MOLECULAR DOCKING STUDIES OF BIS-(THEOPHYLLINATO)-TETRAAQUOCOBALT(II) COMPLEX
}

El Hamdani, Hicham (student, Meknes, MAR); EL Amane, Mohamed (Moulay Ismail University, School of sciences, Meknes, MAR); Duhayon, Carine (CNRS ; LCC (Laboratoire de Chimie de Coordination), toulouse, FRA)

The compound of the general formula: $\left[\mathrm{Co}\left(\mathrm{C}_{7} \mathrm{H}_{8} \mathrm{~N}_{4} \mathrm{O}_{2}\right)_{2}\left(\mathrm{H}_{2} \mathrm{O}\right)_{4}\right]$ has been prepared and characterized by $\mathrm{X}$-ray diffraction analysis, UV-visible and infrared spectroscopy (FTIR), ${ }^{1} \mathrm{H},{ }^{13} \mathrm{C} N \mathrm{NR}$ and thermal analyses (TGA and DTA). The complex was crystallized in the monoclinic system (P 21/c). The unit cell parameters are $a=7.6304$ (3) $\AA, b=$ 13.1897 (6) $\AA, c=9.6670$ (4) $\AA, \beta=104.9744$ (17) ${ }^{\circ}$ and $V=939.87$ (7) $\AA 3$. The cobalt ions are pseudo-octahedrally coordinated by two theophyllinato ligands with Co-N distance is $2.1847 \AA$, and four coordinated water with Co-O distances included between 2.0756 and $2.1022 \AA$. The three-dimensional network stabilized by the intermolecular hydrogen bonds $(\mathrm{O}-\mathrm{H} \ldots \mathrm{O}$ and $\mathrm{O}-\mathrm{H} \ldots \mathrm{N})$ which incorporated $24(8)$ and 22 (18) graph-set motifs. The fingerprint plots associated with the Hirshfeld surface clearly display each significant interaction involved in the structure, by quantifying them in an effective visual manner. Additionally, the molecular docking studies between the tumor suppressor protein $\mathrm{p} 53$ with the cobalt complexes. The results of molecular docking show that the complex has a good affinity complex-P53 (docking score $=7.19$ ). 